\title{
Correction to: Cylindrical Martingale Problems Associated with Lévy Generators
}

\section{David Criens ${ }^{1}$}

Published online: 3 June 2020

(c) Springer Science+Business Media, LLC, part of Springer Nature 2020

\section{Correction to: J Theor Probab (2019) 32:1306-13 https://doi.org/10.1007/s10959-018-0814-4}

\section{Corrections}

In this note, we correct claims made in [2]:

(i) It is claimed that the generalized martingale problem introduced in [2] allows explosion in a continuous manner. However, because the cemetery $\Delta$ is added to $\mathbb{B}$ as an isolated point, explosion can only happen by a jump and is excluded by [2, Lemma 4.3]. In Sect. 2, we explain how the setup can be adjusted to include the possibility of explosion.

(ii) In the proof of [2, Proposition 4.8], it is needed that the operator $A$ has a non-empty resolvent set $\rho(A)$, i.e., that

$$
\rho(A) \triangleq\left\{\lambda \in \mathbb{R}:(\lambda-A)^{-1} \text { exists in } L(\mathbb{B}, \mathbb{B})\right\} \neq \emptyset .
$$

This assumption is missing in [2]. It is, e.g., satisfied in case $A$ is the generator of a $C_{0}$-semigroup; see [4, Remark 1.1.3, Proposition 1.2.1].

\section{A Setup Including Explosion}

\subsection{Modified Setup}

In the following, we explain how $\Omega, \tau_{n}$ and $\tau_{\Delta}$ have to be redefined such that the setting includes the possibility of explosion.

The original article can be found online at https://doi.org/10.1007/s10959-018-0814-4.

$凶$ David Criens

david.criens@tum.de

1 Center for Mathematics, Technical University of Munich, Munich, Germany 
For a function $\omega: \mathbb{R}_{+} \rightarrow \mathbb{B}_{\Delta}$, we define

$$
\tau_{\Delta}(\omega) \triangleq \inf \left(t \in \mathbb{R}_{+}: \omega(t)=\Delta\right)
$$

where, as always, $\inf (\emptyset) \triangleq \infty$. Let $\Omega$ to be the space of all right continuous functions $\omega: \mathbb{R}_{+} \rightarrow \mathbb{B}_{\Delta}$ which are càdlàg on $\left[0, \tau_{\Delta}(\omega)\right)$ and satisfy $\omega(t)=\Delta$ for all $t \geq \tau_{\Delta}(\omega)$. The difference in comparison with the setting in [2] is that $\omega \in\left\{\tau_{\Delta}<\infty\right\}$ might not have a left limit at $\tau_{\Delta}(\omega)$.

Denote by $X$ the coordinate process, i.e., $X_{t}(\omega)=\omega(t)$ for all $\omega \in \Omega$ and $t \in \mathbb{R}_{+}$, and denote by $\mathcal{F} \triangleq \sigma\left(X_{t}, t \in \mathbb{R}_{+}\right)$the $\sigma$-field generated by $X$. The proof of the following is given in Sect. 2.2.

Lemma 1 There exists a metric $d_{\Omega}$ on $\Omega$ such that $\left(\Omega, d_{\Omega}\right)$ is separable and complete and $\mathcal{F}$ is the corresponding Borel $\sigma$-field.

Let $\mathbf{F}=\left(\mathcal{F}_{t}\right)_{t \geq 0}$ be the filtration generated by $X$, i.e. $\mathcal{F}_{t} \triangleq \sigma\left(X_{s}, s \in[0, t]\right)$ for $t \in \mathbb{R}_{+}$. Note that $\tau_{\Delta}$ is an $\mathbf{F}$-stopping time, because $\left\{\tau_{\Delta} \leq t\right\}=\left\{X_{t}=\Delta\right\} \in \mathcal{F}_{t}$. For $\Gamma \subseteq \mathbb{B}$, we define

$$
\tau(\Gamma) \triangleq \inf \left(t<\tau_{\Delta}: X_{t} \in \Gamma \text { or } X_{t-} \in \Gamma\right) \wedge \tau_{\Delta}
$$

The proof of the following is given in Sect.2.3.

Lemma 2 (i) If $\Gamma \subseteq \mathbb{B}$ is closed, then $\tau(\Gamma)$ is an $\mathbf{F}$-stopping time.

(ii) If $\Gamma_{1} \subseteq \Gamma_{2} \subseteq \Gamma_{3} \subseteq \cdots$ is an increasing sequence of open sets in $\mathbb{B}$ such that $\bigcup_{n \in \mathbb{N}} \Gamma_{n}=\mathbb{B}$, then $\tau\left(\mathbb{B} \backslash \Gamma_{n}\right) \nearrow \tau_{\Delta}$ as $n \rightarrow \infty$.

We define

$$
\tau_{n} \triangleq \inf \left(t<\tau_{\Delta}:\left\|X_{t}\right\| \geq n \text { or }\left\|X_{t-}\right\| \geq n\right) \wedge \tau_{\Delta} \wedge n, \quad n \in \mathbb{N} .
$$

By Lemma 2, $\left(\tau_{n}\right)_{n \in \mathbb{N}}$ is a sequence of $\mathbf{F}$-stopping times satisfying $\tau_{n} \nearrow \tau_{\Delta}$ as $n \rightarrow \infty$. In this modified setting, the GMP can be defined as in [2] and all results from [2] hold. In Sect. 3, we comment on necessary changes in the proofs.

\subsection{Proof of Lemma 1}

We adapt the proof of [1, Lemma A.7]. Define

$$
\Omega^{\star} \triangleq\left(D\left(\mathbb{R}_{+}, \mathbb{B}\right) \times(0, \infty]\right) \cup\left(\left\{\omega_{\Delta}\right\} \times\{0\}\right),
$$


where $\omega_{\Delta}(t)=\Delta$ for all $t \in \mathbb{R}_{+}$. For $z \in[0, \infty]$ and $t \in \mathbb{R}_{+}$, we define

$$
\begin{aligned}
\phi_{z}(t) \triangleq \begin{cases}t, & z=\infty, \\
z\left(1-e^{-t}\right), & z \in(0, \infty), \\
0, & z=0,\end{cases} \\
\phi_{z}^{-1}(t) \triangleq \begin{cases}t, & z=\infty, \\
-\log \left(1-\frac{t}{z}\right) \mathbf{1}_{\{t<z\}}, & z \in(0, \infty), \\
0, & z=0 .\end{cases}
\end{aligned}
$$

Moreover, we define $\iota: \Omega \rightarrow \Omega^{\star}$ by

$$
\iota(\omega) \triangleq\left(\omega \circ \phi_{\tau_{\Delta}(\omega)}, \tau_{\Delta}(\omega)\right)
$$

Lemma 3 เ is a bijection.

Proof To check the injectivity, let $\omega, \alpha \in \Omega$ be such that $\iota(\omega)=\iota(\alpha)$. In case $\tau_{\Delta}(\omega)=$ $\tau_{\Delta}(\alpha) \in\{0, \infty\}$, we clearly have $\omega=\alpha$. In case $0<\tau_{\Delta}(\omega)=\tau_{\Delta}(\alpha)<\infty$, we can deduce from the first coordinates of $\iota(\omega)$ and $\iota(\alpha)$ that $\omega=\alpha$ on $\left[0, \tau_{\Delta}(\omega)\right)=$ $\left[0, \tau_{\Delta}(\alpha)\right)$, which implies $\omega=\alpha$.

To check the surjectivity, note that $\iota\left(\omega_{\Delta}\right)=\left(\omega_{\Delta}, 0\right)$ and that $\iota\left(\omega \circ \phi_{t}^{-1} \mathbf{1}_{[0, t)}+\right.$ $\left.\Delta \mathbf{1}_{[t, \infty)}\right)=(\omega, t)$ for all $(\omega, t) \in D\left(\mathbb{R}_{+}, \mathbb{B}\right) \times(0, \infty]$.

Let $d_{D}$ be the Skorokhod metric on $D\left(\mathbb{R}_{+}, \mathbb{B}_{\Delta}\right)$ and let $d_{[0, \infty]}$ be the arctan metric on $[0, \infty]$. We define

$$
d_{D \times[0, \infty]}((\omega, t),(\alpha, s)) \triangleq d_{D}(\omega, \alpha)+d_{[0, \infty]}(t, s)
$$

for $(\omega, t),(\alpha, s) \in D\left(\mathbb{R}_{+}, \mathbb{B}_{\Delta}\right) \times[0, \infty]$, and set

$$
\left.d_{\Omega^{\star}} \triangleq d_{D \times[0, \infty]}\right|_{\Omega^{\star} \times \Omega^{\star}}
$$

We note that $\left(\Omega^{\star}, d_{\Omega^{\star}}\right)$ is separable and complete, because it is a $G_{\delta}$ subspace of $\left(D\left(\mathbb{R}_{+}, \mathbb{B}_{\Delta}\right) \times[0, \infty], d_{D \times[0, \infty]}\right)$. Due to Lemma 3 , we can equip $\Omega$ with the metric

$$
\begin{aligned}
d_{\Omega}(\omega, \alpha) & \triangleq d_{\Omega^{\star}}(\iota(\omega), \iota(\alpha)) \\
& =d_{D}\left(\omega \circ \phi_{\tau_{\Delta}(\omega)}, \alpha \circ \phi_{\tau_{\Delta}(\alpha)}\right)+d_{[0, \infty]}\left(\tau_{\Delta}(\omega), \tau_{\Delta}(\alpha)\right)
\end{aligned}
$$

for $\omega, \alpha \in \Omega$. In this case, $\iota$ is an isometry and $\left(\Omega, d_{\Omega}\right)$ is separable and complete. In the following, we equip $\Omega$ with the topology induced by the metric $d_{\Omega}$.

We now prove that $\mathcal{F}=\mathcal{B}(\Omega)$. By the definition of the metric $d_{\Omega}$, the maps

$$
\Omega \ni \omega \mapsto \omega \circ \phi_{\tau_{\Delta}(\omega)} \in D\left(\mathbb{R}_{+}, \mathbb{B}_{\Delta}\right), \quad \Omega \ni \omega \mapsto \tau_{\Delta}(\omega) \in[0, \infty]
$$


are continuous. For fixed $t \in \mathbb{R}_{+}$, the map $[0, \infty] \ni z \mapsto \phi_{z}^{-1}(t) \in \mathbb{R}_{+}$is Borel and, consequently, also

$$
\Omega \ni \omega \mapsto \phi_{\tau_{\Delta}(\omega)}^{-1}(t) \in \mathbb{R}_{+}
$$

is Borel. Because right continuous adapted processes are progressively measurable, the map

$$
D\left(\mathbb{R}_{+}, \mathbb{B}_{\Delta}\right) \times \mathbb{R}_{+} \ni(\omega, t) \mapsto \omega(t) \triangleq Y(\omega, t) \in \mathbb{B}_{\Delta}
$$

is Borel. We conclude that for every $t \in \mathbb{R}_{+}$the map

$$
\Omega \ni \omega \mapsto \omega(t)=Y\left(\omega \circ \phi_{\tau_{\Delta}(\omega)}, \phi_{\tau_{\Delta}(\omega)}^{-1}(t)\right) \mathbf{1}_{\left\{t<\tau_{\Delta}(\omega)\right\}}+\Delta \mathbf{1}_{\left\{t \geq \tau_{\Delta}(\omega)\right\}} \in \Omega
$$

is Borel. This implies that $\mathcal{F} \subseteq \mathcal{B}(\Omega)$.

Note that $\iota$ is $\mathcal{F} / \mathcal{B}\left(\Omega^{\star}\right)$ measurable. Let $f: \Omega \rightarrow \mathbb{R}$ be a Borel function. Because $\iota$ is an isometry, the inverse map $\iota^{-1}: \Omega^{\star} \rightarrow \Omega$ is continuous and therefore Borel. We conclude that

$$
\Omega \ni \omega \mapsto f(\omega)=\left(\left(f \circ \iota^{-1}\right) \circ \iota\right)(\omega) \in \mathbb{R}
$$

is $\mathcal{F} / \mathcal{B}(\mathbb{R})$ measurable as composition of the $\mathcal{B}\left(\Omega^{\star}\right) / \mathcal{B}(\mathbb{R})$ measurable map $f \circ \iota^{-1}$ and the $\mathcal{F} / \mathcal{B}\left(\Omega^{\star}\right)$ measurable map $\iota$. This implies $\mathcal{B}(\Omega) \subseteq \mathcal{F}$ and the proof is complete.

\subsection{Proof of Lemma 2}

(i). We have to show that $\{\tau(\Gamma) \leq t\} \in \mathcal{F}_{t}$ for all $t \in \mathbb{R}_{+}$. For $x \in \mathbb{B}$, we define $d(x, \Gamma) \triangleq \inf _{y \in \Gamma}\|x-y\|$ and set

$$
\Gamma_{n} \triangleq\left\{x \in \mathbb{B}: d(x, \Gamma)<\frac{1}{n}\right\}
$$

Moreover, on $\left\{t<\tau_{\Delta}\right\}$ we set

$$
F_{t} \triangleq \operatorname{cl}_{\mathbb{B}}\left(\left\{X_{s}: s \in[0, t]\right\}\right)=\left\{X_{s}, X_{s-}: s \in[0, t]\right\} \subseteq \mathbb{B} .
$$

Because $x \mapsto d(x, \Gamma)$ is Lipschitz continuous, the set $\Gamma_{n}$ is open, and because $\Gamma$ is closed, $\Gamma=\{x \in \mathbb{B}: d(x, \Gamma)=0\}$. Define $\tau \triangleq \sup _{n \in \mathbb{N}} \tau\left(\Gamma_{n}\right)$. Because $\Gamma \subseteq \Gamma_{n}$, it is clear that $\tau \leq \tau(\Gamma)$. Next, we show that $\tau \geq \tau(\Gamma)$. We claim that this inequality follows if we show that

$$
\forall t \in \mathbb{R}_{+}: \bigcap_{n \in \mathbb{N}}\left\{F_{t} \cap \Gamma_{n} \neq \emptyset\right\} \subseteq\left\{F_{t} \cap \Gamma \neq \emptyset\right\} \text { on }\left\{t<\tau_{\Delta}\right\}
$$

We explain this: In case $\tau \geq \tau_{\Delta}$, we have $\tau=\tau(\Gamma)=\tau_{\Delta}$. Take $\omega \in\left\{\tau<\tau_{\Delta}\right\}$ and let $\varepsilon>0$ be such that $\varepsilon<\tau_{\Delta}(\omega)-\tau(\omega)$ in case $\tau_{\Delta}(\omega)<\infty$. For each $n \in \mathbb{N}$, we find a $t_{n} \in\left[\tau\left(\Gamma_{n}\right)(\omega), \tau\left(\Gamma_{n}\right)(\omega)+\varepsilon\right)$ such that $F_{t_{n}}(\omega) \cap \Gamma_{n} \neq \emptyset$. Note that $t \triangleq \sup _{n \in \mathbb{N}} t_{n} \leq$ 
$\tau(\omega)+\varepsilon<\tau_{\Delta}(\omega)$ and that $F_{t}(\omega) \cap \Gamma_{n} \neq \emptyset$ for all $n \in \mathbb{N}$. Consequently, in case (2.1) holds we have $F_{t}(\omega) \cap \Gamma \neq \emptyset$, which implies $\tau(\Gamma)(\omega) \leq t \leq \tau(\omega)+\varepsilon$. We conclude that $\tau \geq \tau(\Gamma)$ as claimed. We proceed showing (2.1). Fix $t \in \mathbb{R}_{+}$. Because on $\left\{t<\tau_{\Delta}\right\}$

$$
\bigcap_{n \in \mathbb{N}}\left\{F_{t} \cap \Gamma_{n} \neq \emptyset\right\} \subseteq\left\{\inf _{x \in F_{t}} d(x, \Gamma)=0\right\}
$$

it suffices to show that on $\left\{t<\tau_{\Delta}\right\}$

$$
\left\{\inf _{x \in F_{t}} d(x, \Gamma)=0\right\} \subseteq\left\{F_{t} \cap \Gamma \neq \emptyset\right\}
$$

Take $\omega \in\left\{t<\tau_{\Delta}\right\}$. Because $\{\omega(\cdot \wedge t)\}$ is compact in $D\left(\mathbb{R}_{+}, \mathbb{B}\right), F_{t}(\omega)$ is compact in $\mathbb{B}$ by [4, Problem 16, p. 152]. Consequently, due to its continuity, the function $x \mapsto d(x, \Gamma)$ attains its infimum on $F_{t}(\omega)$. Thus, because $\Gamma=\{x \in \mathbb{B}: d(x, \Gamma)=0\}$, if $\inf _{x \in F_{t}(\omega)} d(x, \Gamma)=0$, we have $F_{t}(\omega) \cap \Gamma \neq \emptyset$. We conclude that (2.1) holds and hence that $\tau=\tau(\Gamma)$.

From the equality $\tau=\tau(\Gamma)$, we deduce that for all $t \in \mathbb{R}_{+}$

$$
\{\tau(\Gamma) \leq t\}=\bigcap_{n \in \mathbb{N}}\left\{\tau\left(\Gamma_{n}\right) \leq t\right\} .
$$

Fix $t \in \mathbb{R}_{+}$and set $\mathbb{Q}_{+}^{t} \triangleq\left([0, t) \cap \mathbb{Q}_{+}\right) \cup\{t\}$. We note that

$$
\begin{aligned}
\left\{\tau\left(\Gamma_{n+1}\right) \leq t<\tau_{\Delta}\right\} & =\bigcap_{m \in \mathbb{N}}\left\{\tau\left(\Gamma_{n+1}\right)<t+\frac{1}{m} \leq \tau_{\Delta}\right\} \\
& \left.\supseteq \bigcup_{s \in \mathbb{Q}_{+}^{t}}\left\{X_{S} \in \Gamma_{n+1}\right\}\right) \cap\left\{t<\tau_{\Delta}\right\} .
\end{aligned}
$$

Because $\Gamma_{n+1}$ is open, we have

$$
\tau\left(\Gamma_{n+1}\right)=\inf \left(t<\tau_{\Delta}: X_{t} \in \Gamma_{n+1}\right) \wedge \tau_{\Delta}
$$

Thus, in case $\tau\left(\Gamma_{n+1}\right) \leq t<\tau_{\Delta}$, the right continuity of $X$ yields that $X_{\tau\left(\Gamma_{n+1}\right)} \in$ $\operatorname{cl}_{\mathbb{B}}\left(\Gamma_{n+1}\right) \subseteq \Gamma_{n}$. We conclude that on $\left\{t<\tau_{\Delta}\right\}$

$$
\left\{\tau\left(\Gamma_{n+1}\right) \leq t\right\} \subseteq \bigcup_{s \in[0, t]}\left\{X_{s} \in \operatorname{cl}_{\mathbb{B}}\left(\Gamma_{n+1}\right)\right\} \subseteq \bigcup_{s \in \mathbb{Q}_{+}^{t}}\left\{X_{s} \in \Gamma_{n}\right\}
$$

Now, (2.2), (2.3) and (2.4) imply that

$$
\left\{\tau(\Gamma) \leq t<\tau_{\Delta}\right\}=\left(\bigcap_{n \in \mathbb{N}_{s} \in \mathbb{Q}_{+}^{t}} \bigcup_{S}\left\{X_{s} \in \Gamma_{n}\right\}\right) \cap\left\{X_{t} \neq \Delta\right\} \in \mathcal{F}_{t} .
$$


Because

$$
\left\{\tau(\Gamma) \leq t, \tau_{\Delta} \leq t\right\}=\left\{\tau_{\Delta} \leq t\right\}=\left\{X_{t}=\Delta\right\} \in \mathcal{F}_{t},
$$

we conclude that $\tau(\Gamma)$ is a stopping time. The proof of (i) is complete.

(ii). Because $n \mapsto \tau\left(\mathbb{B} \backslash \Gamma_{n}\right)$ is increasing, $\tau\left(\mathbb{B} \backslash \Gamma_{n}\right) \nearrow \tau \triangleq \sup _{n \in \mathbb{N}} \tau\left(\mathbb{B} \backslash \Gamma_{n}\right)$ as $n \rightarrow \infty$. Because $\tau \leq \tau_{\Delta}$, it suffices to show that $\tau \geq \tau_{\Delta}$. For contradiction, suppose that there exists an $\omega \in\left\{\tau<\tau_{\Delta}\right\}$ and set $\omega^{\prime} \triangleq \omega(\cdot \wedge \tau(\omega)) \in D\left(\mathbb{R}_{+}, \mathbb{B}\right)$. Then,

$$
\tau\left(\mathbb{B} \backslash \Gamma_{n}\right)\left(\omega^{\prime}\right)=\inf \left(t \in \mathbb{R}_{+}: \omega^{\prime}(t) \notin \Gamma_{n} \text { or } \omega^{\prime}(t-) \notin \Gamma_{n}\right) \nearrow \infty \text { as } n \rightarrow \infty
$$

Because $\tau\left(\mathbb{B} \backslash \Gamma_{n}\right)$ is an F-stopping time by (i), so is $\tau$ and Galmarino's test (see [6, Lemma III.2.43]) implies that $\tau(\omega)=\tau\left(\omega^{\prime}\right)=\infty$. This is a contradiction and $\tau=\tau_{\Delta}$ follows. The proof of (ii) is complete.

\section{Modifications, Corrections and Comments on Proofs}

\section{$3.1[2$, Lemma 4.3]}

The last conclusion in [2, Lemma 4.3] is empty: In the setting of [2], it cannot happen that $X_{\tau_{\Delta}-}=\Delta$.

\section{$3.2[2$, Lemmata 4.3, 4.5]}

Due to the initial value and the possibility that $X$ has no left limit at $\tau_{n}$, some bounds in the proofs of [2, Lemmata $4.3,4.5]$ are only valid on the open stochastic interval $\rrbracket 0, \tau_{n} \llbracket$. Because singletons have Lebesgue measure zero, the arguments require no further changes.

The last conclusion in the proof of [2, Lemma 4.5] follows from the dominated convergence theorem.

\section{$3.3[2$, Proposition 4.8]}

In the proof, it has been used that $\rho\left(A^{*}\right) \neq \emptyset$, see [8, Lemma 4.1]. Because $\mathbb{B}$ is separable and reflexive, its dual $\mathbb{B}^{*}$ is separable and $D$ in the proof of [2, Proposition 4.8] can be constructed more directly: The assumption $\rho(A) \neq \emptyset$ implies that $\rho\left(A^{*}\right) \neq$ $\emptyset$, see [7, Theorem 5.30, p. 169]. Let $D^{\prime} \subset \mathbb{B}^{*}$ be a countable dense subset of $\mathbb{B}^{*}$ and take $\lambda \in \rho\left(A^{*}\right)$. Now, set $R\left(\lambda, A^{*}\right) \triangleq\left(\lambda-A^{*}\right)^{-1}$ and define $D \triangleq\left\{R\left(\lambda, A^{*}\right) x: x \in\right.$ $\left.D^{\prime}\right\} \subseteq D\left(A^{*}\right)$. We claim that for each $x \in D\left(A^{*}\right)$ there exists a sequence $\left(x_{n}\right)_{n \in \mathbb{N}} \subset D$ such that $x_{n} \rightarrow x$ and $A^{*} x_{n} \rightarrow A^{*} x$ in the operator norm as $n \rightarrow \infty$. To see this, take $x \in D\left(A^{*}\right)$ and set $y \triangleq \lambda x+A^{*} x$. There exists a sequence $\left(y_{n}\right)_{n \in \mathbb{N}} \subset D^{\prime}$ such that $y_{n} \rightarrow y$ as $n \rightarrow \infty$. Finally, set $x_{n} \triangleq R\left(\lambda, A^{*}\right) y_{n} \in D$. Because $R\left(\lambda, A^{*}\right) \in$ $L\left(\mathbb{B}^{*}, \mathbb{B}^{*}\right)$, we have $x_{n} \rightarrow R\left(\lambda, A^{*}\right) y=x$ as $n \rightarrow \infty$. Moreover, the triangle 
inequality yields that

$$
\left\|A^{*} x_{n}-A^{*} x\right\| \leq\left\|y_{n}-y\right\|+|\lambda|\left\|x_{n}-x\right\| \rightarrow 0 \text { as } n \rightarrow \infty .
$$

The claim is shown.

\section{$3.4[2$, Lemma 4.10]}

Due to Lemma 1 , it is not necessary to pass to $D\left(\mathbb{R}_{+}, \mathbb{B}_{\Delta}\right)$. Moreover, it can be seen more easily that $\Phi$ is Borel. Indeed, $\Phi$ is continuous.

\section{5 [2, Lemma 4.11]}

In the proof of $P$-a.s.

$$
E^{P}\left[\left(M_{t \wedge \tau_{n}}^{f}-M_{s \wedge \tau_{n}}^{f}\right) \circ \theta_{\xi} \mathbf{1}_{\left\{\xi<\tau_{\Delta}\right\}} \mid \mathcal{F}_{s+\xi}\right]=0
$$

the variable $n$ is used twice, which results in a conflict of notation. We correct the argument: Note that $\tau_{n+k} \circ \theta_{\xi}+\xi \leq \tau_{2(n+k)}$ on $\left\{\xi<\tau_{n+k}\right\}$ for all $k \in \mathbb{N}$. Set $\sigma_{r} \triangleq r \wedge \tau_{n} \circ \theta_{\xi}+\xi$. We obtain that $P$-a.s.

$$
\begin{aligned}
E^{P} & {\left[\left(M_{t \wedge \tau_{n}}^{f}-M_{s \wedge \tau_{n}}^{f}\right) \circ \theta_{\xi} \mathbf{1}_{\left\{\xi<\tau_{\Delta}\right\}} \mid \mathcal{F}_{s+\xi}\right] } \\
& =\lim _{k \rightarrow \infty} E^{P}\left[\left(M_{\sigma_{t}}^{f}-M_{\sigma_{s}}^{f}\right) \mathbf{1}_{\left\{\xi<\tau_{n+k}\right\}} \mid \mathcal{F}_{s+\xi}\right] \\
& =\lim _{k \rightarrow \infty} E^{P}\left[\left(M_{\sigma_{t} \wedge \tau_{2(n+k)}}^{f}-M_{\sigma_{s} \wedge \tau_{2(n+k)}}^{f}\right) \mathbf{1}_{\left\{\xi<\tau_{n+k}\right\}} \mid \mathcal{F}_{s+\xi}\right] \\
& =\lim _{k \rightarrow \infty}\left(M_{\sigma_{t} \wedge \tau_{2(n+k)} \wedge(s+\xi)}^{f}-M_{\sigma_{s} \wedge \tau_{2(n+k)} \wedge(s+\xi)}^{f}\right) \mathbf{1}_{\left\{\xi<\tau_{n+k}\right\}}=0,
\end{aligned}
$$

by the optional stopping theorem.

\section{$3.6[2$, Section 4.3.2]}

Because $X$ has no left limit at $\tau_{\Delta}$, the random measure $\mu^{X}$ cannot be defined as in [2, Eq. 4.20]. We pass to a stopped version: Let $\widehat{X}$ be defined as in Eq. 4.11 in [2] and set $X^{n} \triangleq \widehat{X}_{\cdot \wedge \tau_{n}}$ and

$$
\begin{aligned}
& \mu^{n}(\omega ; d t, d x) \triangleq \sum_{s>0} \mathbf{1}_{\left\{\Delta X_{s}^{n}(\omega) \neq 0\right\}} \varepsilon_{\left(s, \Delta X_{s}^{n}(\omega)\right)}(d t, d x) \\
& v^{n}(\omega ; d t, d x) \triangleq \mathbf{1}_{\left\{t \leq \tau_{n}(\omega)\right\}} K\left(X_{t}^{n}(\omega), d x\right) d t
\end{aligned}
$$

We have the following version of [2, Lemmata 4.17, 4.18, 4.19]:

Lemma 4 Forall $n \in \mathbb{N}$ the random measure $\mu^{n}$ is $\left(\mathbf{F}^{P}, P\right)$-optional with $\mathscr{P}^{P}$ - $\sigma$-finite Doléans measure and $\left(\mathbf{F}^{P}, P\right)$-predictable compensator $v^{n}$. 
Because the proofs of [2, Lemmata 4.17, 4.18] contain typos and the proof of [2, Lemma 4.19] requires some minor modification, as the set $\mathcal{Z}_{1} \times \mathcal{Z}_{2}$ has not all claimed properties, we give a proof:

Proof Due to [3, Theorem IV.88B, Remark below], the set $\left\{\Delta X^{n} \neq 0\right\}$ is $\mathbf{F}^{P}$-thin. Hence, [6, II.1.15] yields that $\mu^{n}$ is $\mathbf{F}^{P}$-optional. It follows as in [9, Example 2, pp. $160]$ that $M_{\mu^{n}}^{P}$ is $\mathscr{P}^{P}-\sigma$-finite. Next, we show that $v^{n}$ is $\mathbf{F}^{P}$-predictable with $\mathscr{P}^{P}$ $\sigma$-finite Doléons measure $M_{v^{n}}^{P}$. For $m \in \mathbb{N}$ we set $G_{m} \triangleq\left\{x \in \mathbb{B}:\|x\| \geq \frac{1}{m}\right\} \cup\{0\}$. Let $W$ be a nonnegative $\mathscr{P}^{P} \otimes \mathscr{B}(\mathbb{B})$-measurable function which is bounded by a constant $c>0$. Because $P$-a.s.

$$
W \mathbf{1}_{\llbracket 0, \tau_{m} \rrbracket} \mathbf{1}_{G_{m}} \star v_{\infty}^{n} \leq \mathrm{cm} \sup _{\|x\| \leq m} K\left(x,\left\{z \in \mathbb{B}:\|z\| \geq \frac{1}{m}\right\}\right)<\infty
$$

we conclude that $M_{v^{n}}^{P}$ is $\mathscr{P}^{P}-\sigma$-finite. Furthermore, the process

$$
W \star \nu^{n}=\lim _{m \rightarrow \infty} W \mathbf{1}_{\left[0, \tau_{m}\right]} \mathbf{1}_{G_{m}} \star \nu^{n}
$$

is $\mathbf{F}^{P}$-predictable as the pointwise limit of an $\mathbf{F}^{P}$-predictable process. We conclude that $v^{n}$ is an $\mathbf{F}^{P}$-predictable random measure.

It remains to show that $v^{n}$ is the $\left(\mathbf{F}^{P}, P\right)$-predictable compensator of $\mu^{n}$. Let $\mathcal{Z}_{1}$ be the collection of sets $A \times\{0\}$ for $A \in \mathcal{F}_{0}^{P}$ and $\llbracket 0, \xi \rrbracket$ for all $\mathbf{F}^{P}$-stopping times $\xi$, and let $\mathcal{Z}_{2}$ be the collection of all sets

$$
G \triangleq\left\{x \in \mathbb{B}:\left(\left\langle x, y_{1}^{*}\right\rangle, \ldots,\left\langle x, y_{d}^{*}\right\rangle\right) \in A\right\} \in \mathcal{B}(\mathbb{B})
$$

for $A \in \mathcal{B}\left(\mathbb{R}^{d}\right), y_{1}^{*}, \ldots, y_{d}^{*} \in D\left(A^{*}\right)$ and $d \in \mathbb{N}$. Note that $M_{\mu^{n}}^{P}(A \times\{0\} \times G)=$ $M_{v^{n}}^{P}(A \times\{0\} \times G)=0$ for all $A \in \mathcal{F}_{0}^{P}$ and $G \in \mathcal{B}(\mathbb{B})$. Fix an $\mathbf{F}^{P}$-stopping time $\xi$ and the cylindrical set $G$ given by (3.1). Denote $Y^{n} \triangleq\left(\left\langle X^{n}, y_{1}^{*}\right\rangle, \ldots,\left\langle X^{n}, y_{d}^{*}\right\rangle\right)$. By [2, Lemma 4.7], we obtain

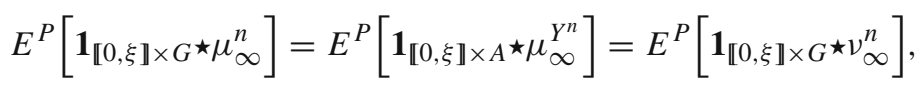

which implies $M_{\mu^{n}}^{P}=M_{v^{n}}^{P}$ on $\mathcal{Z}_{1} \times \mathcal{Z}_{2}$. Take a norming sequence $\left(x_{m}^{*}\right)_{m \in \mathbb{N}} \subset \mathbb{B}^{*}$ of unit vectors, see p. 522 in [5] for a definition, and note that

$$
B_{m} \triangleq\left\{x \in \mathbb{B}:\|x\|>\frac{1}{m}\right\}=\bigcup_{k \in \mathbb{N}}\left\{x \in \mathbb{B}:\left|\left\langle x, x_{k}^{*}\right\rangle\right|>\frac{1}{m}\right\} .
$$

For $m, k \in \mathbb{N}$ set

$$
\gamma(m, k) \triangleq \inf \left(t \in \mathbb{R}_{+}: \mu^{n}\left([0, t] \times B_{m}\right)>k\right) \wedge m
$$

The dominated convergence theorem yields that

$$
M_{\mu^{n}}^{P}\left((A \times B) \cap\left(\llbracket 0, \gamma(m, k) \rrbracket \times B_{m}\right)\right)=M_{v^{n}}^{P}\left((A \times B) \cap\left(\llbracket 0, \gamma(m, k) \rrbracket \times B_{m}\right)\right)
$$


for all $A \times B \in \mathcal{Z}_{1} \times \mathcal{Z}_{2}$. Now, we conclude from the uniqueness theorem for measures that $M_{\mu^{n}}^{P}=M_{v^{n}}^{P}$ on the trace $\sigma$-field $\left(\mathscr{P}^{P} \otimes \mathcal{B}(\mathbb{B})\right) \cap\left(\llbracket 0, \gamma(m, k) \rrbracket \times\left(B_{m} \cup\{0\}\right)\right)$. Finally, taking $k, m \rightarrow \infty$ and using the monotone convergence theorem show that $M_{\mu^{n}}^{P}=M_{v^{n}}^{P}$ on $\mathscr{P}^{P} \otimes \mathcal{B}(\mathbb{B})$. The proof is complete.

The candidate density process $Z$ can be defined as in [2, Lemma 4.21] with $\mu^{X}$ and $v^{X}$ replaced by $\mu^{n}$ and $\nu^{n}$.

\section{$3.7[2$, Lemmata $4.21,4.22]$}

In the proofs, the process $X$ should be replaced by $\widehat{X}$.

\section{8 [2, Proposition 3.7]}

The representation of the CMG densities and the function $V^{k}$ in [2, Lemma 4.23] should be multiplied by $\mathbf{1}_{\left\{\tau_{n}<\tau_{\Delta}\right\}}$. Moreover, in all Lebesgue integrals $X_{-}$should be replaced by $X$.

\section{$3.9[2$, Lemma 3.16]}

Instead of the Yamada-Watanabe argument, the uniqueness also follows from the observation that for a pseudo-contraction semigroup $\left(S_{t}\right)_{t \geq 0}$ and a square integrable Lévy process $L$ the law of $\int_{0}^{*} S{ }_{-s} d L_{s}$ is completely determined by $L$. This can be seen with the approximation argument used in the proof of [11, Theorem 9.20].

\section{Final Comment}

Above [2, Proposition 3.9] it is noted that "in a non-conservative setting, one can try to conclude existence from an extension argument in a larger path space, [but] in this case one has to prove that the extension is supported on $(\Omega, \mathcal{F})$ " as defined in [2]. The larger path space, to which this comment refers, is the path space defined in this correction note. In our modified setting, it follows from Parthasarathy's extension theorem (see [10]) that under the assumptions imposed in [2] the GMP $\left(A, b^{\prime}, a, K^{\prime}, \eta, \tau_{\Delta^{-}}\right)$has a solution whenever the GMP $\left(A, b, a, K, \eta, \tau_{\Delta}-\right)$ has a solution. This observation extends [2, Theorem 3.6].

\section{References}

1. Biagini, S., Bouchard, B., Kardaras, C., Nutz, M.: Robust fundamental theorem for continuous processes. Math. Finance 27(4), 963-987 (2017)

2. Criens, D.: Cylindrical martingale problems associated with Lévy generators. J. Theor. Probab. 32(3), 1306-1359 (2019)

3. Dellacherie, C., Meyer, P.: Probabilities and Potential. North-Holland, Amsterdam (1978)

4. Ethier, S., Kurtz, T.: Markov Processes: Characterization and Convergence. Wiley, Hoboken (2005) 
5. Hytönen, T., van Neerven, J., Veraar, M., Weis, L.: Analysis in Banach Spaces: Volume I: Martingales and Littlewood-Paley Theory. Springer, Berlin (2016)

6. Jacod, J., Shiryaev, A.: Limit Theorems for Stochastic Processes, 2nd edn. Springer, Berlin (2003)

7. Kato, T.: Perturbation Theory for Linear Operators, 2nd edn. Springer, Berlin (1980)

8. Kunze, M.: On a class of martingale problems on Banach spaces. Electron. J. Probab. 18(104), 1-30 (2013)

9. Liptser, R., Shiryaev, A.N.: Theory of Martingales. Springer, Berlin (1989)

10. Parthasarathy, K.: Probability Measures on Metric Spaces. Academic Press, New York (1967)

11. Peszat, S., Zabczyk, J.: Stochastic Partial Differential Equations with Lévy Noise: An Evolution Equation Approach. Cambridge University Press, Cambridge (2007)

Publisher's Note Springer Nature remains neutral with regard to jurisdictional claims in published maps and institutional affiliations. 